\title{
Effect of Novel Watermelon Mouthwash on Oral Micro Flora in Patients Undergoing Orthodontic Treatment
}

\author{
Aravinthrajkumar Govindaraj ${ }^{1}$, Saravana Dinesh S.P2 \\ ${ }^{1}$ Department of Orthodontics, Saveetha Dental College and Hospitals, Chennai, Tamil Nadu, India. \\ ${ }^{2}$ Department of Orthodontics, Saveetha Dental College and Hospitals, Chennai, Tamil Nadu, India.
}

\section{ABSTRACT}

\section{BACKGROUND}

Patients undergoing orthodontic treatment undergo various changes in their oral microbiological profile, which are attributed to the accumulation of plaque and calculus around various orthodontic attachments. Plaque and calculus are primarily composed of various salivary mineral particles and bacteria such as Lactobacillus and Streptococcus mutans. These changes in the oral cavity will result in the formation of white spot lesions around the bracket surface. The aim of this research was to study the antibacterial effect of watermelon mouthwash on Lactobacillus and Streptococcus mutans.

\section{METHODS}

This is an in-vitro study to determine the antibacterial activity of watermelon mouth wash against Lactobacillus and Streptococcus mutans. Fresh cut watermelon is used to prepare the watermelon mouth wash. The extract of watermelon is concentrated by boiling and mixed with various additives to prepare the mouthwash. The antibiotic sensitivity is studied using well diffusion methods in culture plates under three different concentrations [50\%, 100\% and 150\%]. After an incubation period of 24 hours, antibiotic sensitivity is studied by measuring the area of inhibition.

\section{RESULTS}

Area of inhibition was seen in all three different concentrations against both Lactobacillus [50 \% - $20 \mathrm{~mm}, 100 \%-23 \mathrm{~mm}$ and $150 \%-29 \mathrm{~mm}$ ] and Streptococcus mutans [50 \% - $10 \mathrm{~mm}, 100 \%-15 \mathrm{~mm}$ and $150 \%-18 \mathrm{~mm}]$.

\section{CONCLUSIONS}

The antibacterial effect of the novel watermelon mouthwash showed that there was significant activity of the mouthwash against Lactobacillus and Streptococcus mutans. The antibacterial activity was more significant against Lactobacillus than Streptococcus mutans. To support the in vitro results, planned clinical trials will be conducted to study the effect of the watermelon mouth wash against oral microflora in patients undergoing orthodontic treatment.

\section{KEY WORDS}

Watermelon Mouthwash, Natural Mouthwash, Streptococcus mutans, Lactobacillus sp., Oral Microflora
Corresponding Author:

Dr. Aravinthrajkumar Govindaraj.

Department of Orthodontics,

Saveetha Dental College and Hospitals,

No.162, Poonamallee high road,

Velappanchavadi - 600077

Chennai, Tamil Nadu.

E-mail: draravinthrajkumar.sav@gmail.com

DOI: $10.14260 / \mathrm{jemds} / 2020 / 582$

How to Cite This Article:

Govindaraj A, Dinesh SPS. Effect of novel watermelon mouthwash on oral micro flora in patients undergoing orthodontic treatment. J Evolution Med Dent Sci 2020;9(37):2674-2679, $10.14260 / \mathrm{jemds} / 2020 / 582$

Submission 12-06-2020, Peer Review 07-08-2020, Acceptance 12-08-2020, Published 14-09-2020.

Copyright (c) 2020 JEMDS. This is an open access article distributed under Creative Commons Attribution License [Attribution 4.0 International (CC BY 4.0)] 


\section{BACKGROUND}

Patients undergoing orthodontic treatment undergo various changes in their oral microbiological profile which is mainly due to accumulation of plaque and calculus around various orthodontic attachments. Plaque and calculus are primarily composed of various salivary mineral particles and bacteria such as Lactobacillus and Streptococcus mutans. Various components of fixed orthodontic treatment such as Brackets, Bands, Wires, Modules, elastomeric chains, etc., harbour food substances which become difficult for the patient to selfcleanse, thus leading to alteration in the microbiological colony. Fixed or removable orthodontic appliances impede the maintenance of oral hygiene and result in plaque accumulation. Plaque retention surrounding orthodontic appliances leads to enamel demineralization caused by organic acids produced by bacteria in the dental plaque. ${ }^{1}$

The accumulation of food substances around the brackets becomes difficult to be self-cleansed by regular methods of oral hygiene maintenance such as the use toothbrush, mouthwash, dental floss and / or interdental brushes. Even the storage of toothbrush plays a major role in altering the bacterial content in the oral cavity. A study on the contamination of tooth brush among the dental patients and students was studies earlier, which showed that there was accumulation of another bacterium which was not often found in the oral cavity. This can show that the storage of the toothbrush plays a major role in the same. They also showed that there was no significant difference in the bacterial colony count between the dental students and patients which showed that there was lack of awareness about the same between both the groups. ${ }^{2}$

Literature reports that orthodontic treatment induces changes in the oral environment by increasing the number of retentive surfaces for plaque, augmenting the bacterial levels of Streptococcus mutans [SM] and Lactobacillus; these modify the patient's salivary characteristics, for example, $\mathrm{pH}$, buffer capacity, and salivary flow. ${ }^{3-5}$ Lactobacillus is an acidogenic bacteria which grows in acidic $\mathrm{pH}$ and is responsible for the progression of dental caries in orthodontic patients. ${ }^{6}$ These alterations increases the risk in formation of dental caries in some patients which develop in the form of white spots on the enamel surface and generalized gingival alterations that appear at the completion of and even during orthodontic treatment.7,8

Prevention of white spot lesions is an important concern for orthodontists. Furthermore, the material of the bracket could play a role in the degree of bacterial adhesion and plaque accumulation as well as in the risk of caries development. J Van et al in their study stated that the initial affinity of bacteria to solid surfaces is due mostly to electrostatic and hydrophobic interactions. Surfaces with high surface free energy more easily attract bacteria such as S. mutans. ${ }^{9}$ Another study by Eliades et al proved that stainless steel presented the highest critical surface tension and can be expected to have a higher plaque retaining capacity..$^{10}$

Due to increased duration of orthodontic treatment the use of regular mouthwashes such as chlorhexidine will not be advised due it its long-term adverse effects. This brings about the need for new natural or herbal mouthwash which has a significant antibacterial property against oral bacterium and least side effects possible. India being a sub-tropical country we decided to use watermelon which has a significant antibacterial property and available during all times as an active ingredient for the formulation of a new mouthwash. The aim of this research is to study the antibacterial effect of novel watermelon (Citrullus lanatus) mouthwash on common oral bacteria such as Lactobacillus and Streptococcus mutans.

\section{METHODS}

100 gm of fresh cut Citrullus lanatus (watermelon) was used for this research Streptococcus mutans (Figure 1). A mortar and pestle were used to get the watermelon extract (Figure 2). $100 \mathrm{gms}$ of the fruit gave $80 \mathrm{~mL}$ of fresh extract of watermelon (Figure 3). The watermelon extract is concentrated into half of its amount in volume by boiling method (Figure 4). The extract was transferred to a conical flask and boiled to reduce the extract into its $50 \% .5 \mathrm{~mL}$ of the freshly prepared concentrated fruit extract is taken in a test tube. $2.5 \mathrm{~mL}$ of water is added to it as a solvent. $2.5 \mathrm{~mL}$ of ethanol is added to the same which acts as a preservative for the mouthwash. 0.1 $\mathrm{mL}$ of peppermint oil acts as an essential oil along with sucrose which acts as a sweetening agent. ${ }^{11}$

\section{Antibiotic Sensitivity}

The prepared watermelon mouth wash is incorporated into discs in three different concentrations: 50, 100 and 150 percentage by volume. Freshly prepared broth is poured onto the culture plates which after solidification were autoclaved. It was later used for the culture of the bacteria. Lactobacillus and Streptococcus mutans were selected as they are the most common bacteria seen in the patients undergoing orthodontic treatment. Freshly prepared Colonies of Lactobacillus and Streptococcus mutans were allowed to grow in the new media. Wells were cut in the culture media [well diffusion method] and the discs were incorporated into the wells.

The culture plates were incubated in hot air oven at 37 degree Celsius for 24 hours. After the incubation period the culture plates were examined for antibiotic sensitivity.

\section{RESULTS}

After the incubation period of 24 hours, the culture plates were examined for antibiotic sensitivity. The antibacterial sensitivity was determined by measuring the area of inhibition using a ruler. The area of inhibition measured for the effect of watermelon mouthwash against Lactobacillus and Streptococcus mutans is given in the table below. [Table 1]

\begin{tabular}{|cccc|}
\hline Concentrations of Mouthwash & $\mathbf{5 0 \%}$ & $\mathbf{1 0 0} \%$ & $\mathbf{1 5 0} \%$ \\
Lactobacillus & $20 \mathrm{~mm}$ & $22 \mathrm{~mm}$ & $29 \mathrm{~mm}$ \\
Streptococcus mutans & $10 \mathrm{~mm}$ & $15 \mathrm{~mm}$ & $18 \mathrm{~mm}$ \\
\hline
\end{tabular}

Area of inhibition for the effect of watermelon mouthwash against Lactobacillus and Streptococcus mutans in three different concentrations. The culture plates after the period of incubation examined for antibiotic sensitivity against Lactobacillus sp and Streptococcus mutans are shown in the Figure 5 and Figure 6 respectively. 

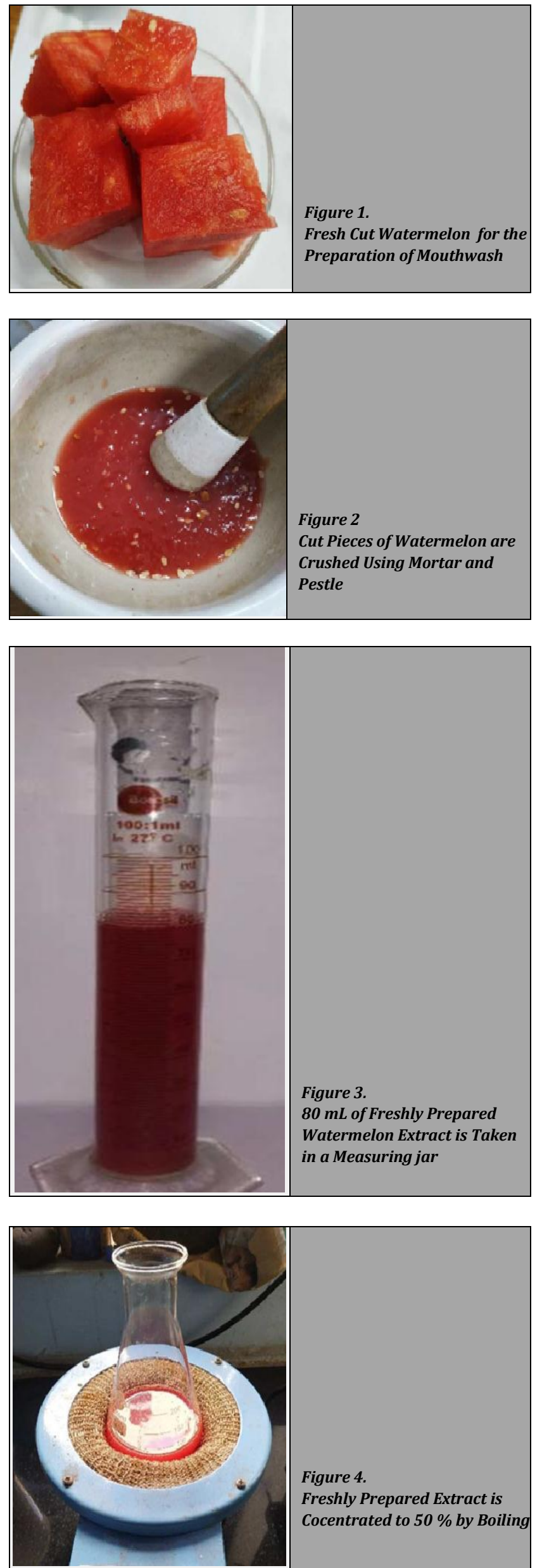

Figure 4.

Freshly Prepared Extract is Cocentrated to $50 \%$ by Boiling
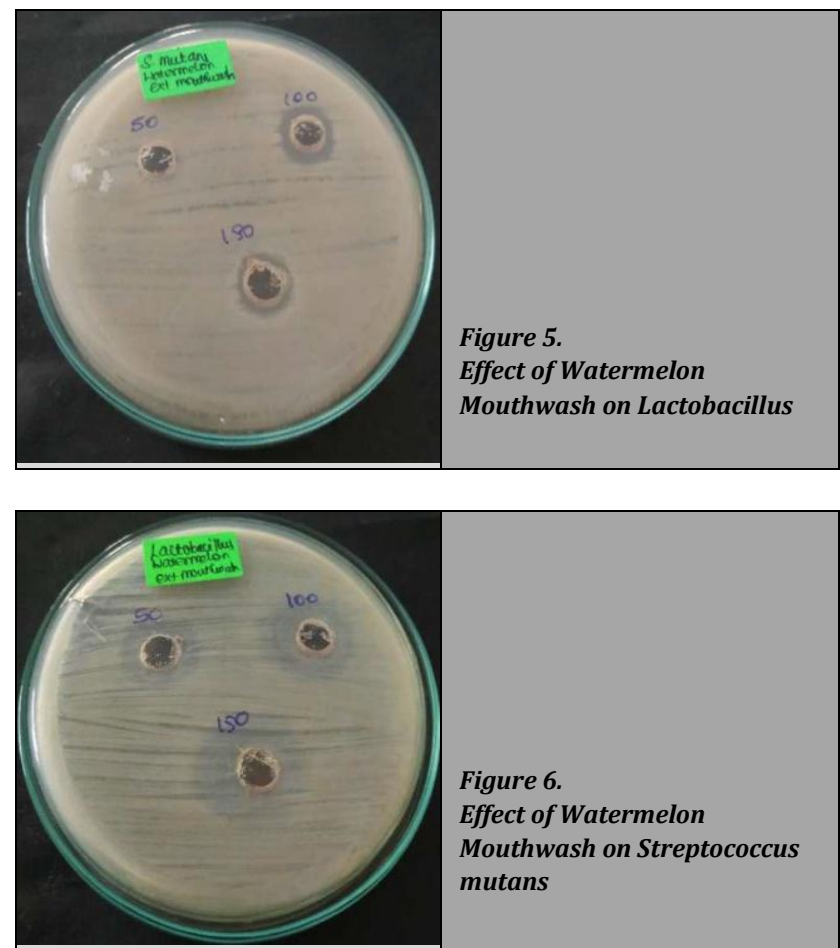

\section{DISCUSSION}

From the results of this study it can be seen that watermelon mouthwash has an innate antibacterial property against Lactobacillus and Streptococcus mutans. The effect of watermelon juice was studied earlier in an invitro study which showed that there was a significant antibacterial effect of the extract against oral microflora. ${ }^{12}$ The antibacterial property of watermelon extract particularly against Lactobacillus was also studied which showed that there was significant effect of watermelon against Lactobacillus even with or without the presence of sucrose in it. The study also showed that the juice along with added sucrose had a significant antibacterial activity when compared to the control group. But there was no active control group in that against common antibacterial mouthwashes. ${ }^{13}$ The phytochemical property of watermelon has not been proven yet which will be a potential field of research in the future.

Lactobacillus and Streptococcus mutans are the most common microflora in the patients undergoing orthodontic treatment which was proven by Lundstrom $F$ et al and Mummolo $S$ et al.14,15 This was the reason for studying the effect of this novel mouthwash against Lactobacillus and Streptococcus mutans in this particular study.

Davis et al showed in his study that adults may experience fewer negative periodontal effects than children and adolescents, provided they are periodontally stable when orthodontic therapy is initiated. Careful consideration of daily oral hygiene, regular maintenance, and monitoring of periodontal status to maintain stability are critical in successful orthodontic treatment in adult populations. ${ }^{16}$ Soncransky et $\mathrm{al}^{17}$ and Van Gastel $\mathrm{J}$ et al ${ }^{18}$ stated that orthodontic appliances encourage plaque accumulation and the development of gingivitis. Study by Karkhanechi et al compared between various fixed orthodontic appliance and removable aligners. The study stated that the use of removable 
aligners facilitates oral hygiene. Treatment with removable aligners was associated with improved periodontal status as evidenced by decreased plaque levels, gingival inflammation, bleeding upon probing, probing pocket depths, and BANA scores. These results suggest that removable aligners be considered when treatment planning for the adult orthodontic patient at risk for periodontitis. ${ }^{19}$ Bands, brackets, and orthodontic wires present unique challenges for hygiene, as these appliances create many food and plaque traps. The areas of the food traps becomes difficult for the human hands to reach through regular oral hygiene practices such as using a toothbrush. ${ }^{18-21}$

The effect of orthodontic appliance on saliva and bacterial changes has been studied earlier. The stimulated salivary flow initially increased after placing orthodontic appliances and subsequently, it diminished, and returned to basal levels. The study also stated that the increase in retentive surfaces increased bacterial levels, leading to increased plaque acidity, and gingival damage rose, as evaluated by occult blood in saliva. $^{21}$

Study by Sharma et al studied correlation of orthodontic treatment by fixed and myofunctional appliance with periodontitis. They stated that there was correlation between malocclusion and periodontitis. Malocclusion leads to periodontitis. Malocclusion is the main reason for the development of poor periodontal health. It may be either due to accumulation of food substances around the crowded teeth surfaces or may be due to the any trauma from occlusion which occurs as a result of the underlying malocclusion. Combined effort has to be played by both periodontist and orthodontist for the treatment of various orthodonticperiodontal problems. ${ }^{22}$

The results of a study by Ireland et al suggested that molar bonds are more likely to elicit changes in the normal plaque microbiota toward a microflora level with raised disease potential. There was a constant increase in levels of microbial species not present at the start of treatment, and not present in plaque associated with molar bands. Although those results do not necessarily indicate that subjects may be more prone to gingivitis or periodontal diseases later in life, there should be concern over the potential for extended-term clinical effects associated with these microbiological changes. ${ }^{23}$

The effects of orthodontic treatment on gingival and periodontal pathogens were studied by various authors. Kim et al in his study showed that during orthodontic leveling and alignment, the frequency of Tannerella forsythia, Campylobactor rectus, and Prevotella nigrescens in gingival crevices increase significantly, thereby heightening the risk of periodontitis and the convention oral hygiene measures performed by patients in that particular study were not able to overcome the development of a more periodonto-pathogenic flora in the gingival sulcus. ${ }^{24}$

Study by Papageorgiou et al elicited the effect of fixed orthodontic appliance on subgingival microbiota after orthodontic treatment and stated that there was increased subgingival prevalence of Aggregatibacter actinomycetemcomitans and Tannerella forsythia in orthodontic patients up to 6 months after appliance removal compared to untreated patients. Insertion of orthodontic fixed appliances seems to be associated with a qualitative change of subgingival microbiota, which reverts to some extent back to normal in the first months after appliance removal. ${ }^{25}$ This is agreeable to the results from the earlier studies which showed that there was significant alterations in the oral microflora colonies in orthodontic patients.

An in vitro study on the antibacterial activity of the green and red fruit extracts of Antidesma bunius on oral pathogenic bacteria showed that there was significant inhibitory effect of the extract against oral pathogenic bacteria. ${ }^{26}$ various studies have been done on the effect of natural and herbal products on oral bacteria. Another study on the antibacterial and antihalitosis effect of fruit extracts especially pomegranate extract was done invitro which showed that the Pomegranate and the Jucá extracts showed higher levels of polyphenols in their phytochemical content, $7.3 \%$ and $3.9 \%$, respectively, and less volatile sulphur compound formation as compared to the control group. The study also stated that, the extracts showed significant antimicrobial activity against the tested oral microorganisms. ${ }^{27}$

A study showing the comparison of chlorhexidine and probiotics has stated that probiotics are as effective as chlorhexidine and can be used as an adjunct in chemical plaque control in patients undergoing orthodontic treatment. Both the chlorhexidine and the probiotic groups showed significant reduction in the values of plaque indices when compared with the control group at the end of the study period. ${ }^{28}$

Another study on the effect of propolis mouthwash in orthodontic patients showed that using Propolis mouthwash had a favourable effect on the gingival health of patients undergoing fixed orthodontic treatment. It also showed that there is an improvement in the gingival, plaque and community periodontal index and the results were comparable to that of chlorhexidine. The study had its own limitations which include that these indices were mostly subjective and interobserver reliability was not assessed for the same which questions the reliability of the study. ${ }^{29}$

Lactobacillus and Streptococcus mutans are the common bacterium which are responsbible for the initiation and progression of caries. These carious lesion in earlier stages might appear in the form of Whitespot lesions in the patients undergoing orthodontic treatment. White spot lesions appear due to an interaction that occurs between bacteria and dietary carbohydrate found in saliva. This results in imbalance in the process of demineralization and remineralization which takes place on the enamel surface and thus resulting in the formation of white spot lesion. ${ }^{30}$

Orthodontic patients are more prone for the formation of white spot lesions. This is due to accumulation of plaque and calculus around the brackets and various orthodontic auxiliaries such as bands, wires, etc. The accumulation of plaque and food substances will result in alteration of the oral $\mathrm{pH}$ which will result in the formation of the bacterial colonies around the surfaces of the orthodontic brackets.

Various treatment modalities have been developed for the prevention and management of white spot lesions in the patients undergoing orthodontic treatment which includes the use of varnishes ${ }^{31}$ such as chlorhexidine or fluoride varnish, ${ }^{32}$ use of remineralizing agents such as CPP-APP, or other restorative treatments.

Chlorhexidine varnishes has a direct effect against the bacteria responsible for formation and progression of dental caries and which appear in the form of white spot lesions. Fluoride varnishes has a remineralizing effect on the enamel 
which will result in the formation of fluorapatite crystals, which in turn will prevent further demineralisation. Fluoride has an additional antibacterial property apart from its remineralizing effect on the enamel. Fluoride varnish containing CPP-ACP had good clinical success in reducing S. mutans count. Use of fluoride varnish containing CPP-ACP and xylitol as a preventive intervention is effective in preventing caries in children. ${ }^{33}$

A study was done regarding the perceptions of the orthodontic patients, parents and the orthodontists on the development of white spot lesions during orthodontic treatment. The orthodontists, general dentists, patients and parents had similar perceptions regarding the prevention, significance and treatment of white spot lesions. All the four groups stated that the patients were the most responsible for the prevention of WSL's. The communication among the patients, their parents, the orthodontists, and the general dentists should have to be improved to reduce the incidence of white spot lesions in the patients undergoing orthodontic treatment. 34

There are various antibacterial mouthwashes available in the market. Chlorhexidine which is considered to be a standard for its effect against oral microflora, has its own side effects on a long-term use. This becomes an important thing to be noted in patients undergoing orthodontic treatment which on average will take from 1.5 to 2 years for completion.

The side effects may affect mostly the oral cavity as discolouration of teeth and soft tissues, loss of taste sensation, numbness, etc. This brings about the need in an alternative for the same with an effective antibacterial property and very negligible side effects in the oral cavity. The current study is an invitro study and clinical studies on the effect of watermelon mouthwash and its phytochemical constituents on orthodontic patients will be published.

Further studies on the effect of watermelon extract and the varnish made from these extracts on white spot lesions around the brackets in patients undergoing orthodontic treatment is in progress and the results of the same will be published in the future.

\section{CONCLUSIONS}

Watermelon mouthwash has antibacterial property against oral microflora particularly Lactobacillus and Streptococcus mutans. The antibacterial activity was more significant against Lactobacillus than Streptococcus mutans. Further well-planned clinical trials are needed to confirm the effect of the mouthwash against oral microflora in patients undergoing orthodontic treatment which will be done in the future studies.

Financial or Other Competing Interests: None.

\section{REFERENCES}

[1] Türkkahraman H, Sayın MO, Bozkurt FY, et al. Archwire ligation techniques, microbial colonization and periodontal status in orthodontically treated patients. Angle Orthod 2005;75(2):231-6.
[2] Govindaraj A, Gurunathan D. Comparision of contamination of tooth brush among dental students and patients. Indian J Public Health Res Development 2019;10(8):28-33.

[3] Chang HS, Walsh LJ, Freer TJ. The effect of orthodontic treatment on salivary flow, $\mathrm{pH}$, buffer capacity and levels of mutans streptococci and lactobacilli. Aust Orthod J 1999;15(4):229-34.

[4] Papaioannou W, Gizani S, Nassika M, et al. Adhesion of Streptococcus mutans to different types of brackets. Angle Orthod 2007;77(6):1090-5.

[5] Yu JK, Ahn SJ, Lee SJ, et al. Quantitative analysis of mutans streptococci adhesion to various orthodontic bracket materials in vivo. Korean J Orthod 2009;39(2):105-11.

[6] Choi JH. Comparison of slim bristled and V-shaped orthodontic toothbrushes in patients with fixed orthodontic appliances. Korean J Orthod 2009;39(6):38392.

[7] Faltermeier A, Bürgers R, Rosentritt M. Bacterial adhesion of Streptococcus mutans to esthetic bracket materials. Am J Orthod Dentofacial Orthop 2008;133(4 Suppl):S99-103.

[8] Alves PVM, Alviano WS, Bolognese AM, et al. Treatment protocol to control Streptococcus mutans level in an orthodontic patient with high caries risk. Am J Orthod Dentofacial Orthop 2008;133(1):91-4.

[9] Dijk JV, Herkströter F, Busscher H, et al. Surface-free energy and bacterial adhesion. An in vivo study in beagle dogs. J Clin Periodontol 1987;14(5):300-4.

[10] Eliades T, Eliades G, Brantley WA. Microbial attachment on orthodontic appliances: I. wettability and early pellicle formation on bracket materials. Am J Orthod Dentofacial Orthop 1995;108(4):351-60.

[11] Ristic M, Svabic MV, Sasic M, et al. Clinical and microbiological effects of fixed orthodontic appliances on periodontal tissues in adolescents. Orthod Craniofac Res 2007;10(4):187-95.

[12] Aravinthrajkumar G, Dinesh SS, Dharan NM. Effect of watermelon juice against oral microflora - an in vitro study. Final Presentation ID: 1013. London, England: IADR/PER General Session 2018.

[13] Govindaraj A, Dinesh SPS, Muralidharan NP. Efficiency of watermelon extract against oral microflora particularly Lactobacillus - an in vitro study. Drug Invention Today 2019;11(1):1-5.

[14] Lundström F, Krasse B. Streptococcus mutansand lactobacilli frequency in orthodontic patients; the effect of chlorhexidine treatments. Eur J Orthod 1987;9(1):10916.

[15] Mummolo S, Tieri M, Nota A, et al. Salivary concentrations of Streptococcus mutans and lactobacilli during an orthodontic treatment. An observational study comparing fixed and removable orthodontic appliances. Clin Exp Dent Res 2020;6(2):181-7.

[16] Davis SM, Plonka AB, Fulks BA, et al. Consequences of orthodontic treatment on periodontal health: clinical and microbial effects. Semin Orthod 2014;20(3):139-49.

[17] Socransky SS, Haffajee AD. Periodontal microbial ecology. Periodontol 2000 2005;38(1):135-87.

[18] Gastel JV, Quirynen M, Teughels W, et al. The relationships between malocclusion, fixed orthodontic appliances and periodontal disease. A review of the literature. Aust Orthod J 2007;23(2):121-9. 
[19] Karkhanechi M, Chow D, Sipkin J, et al. Periodontal status of adult patients treated with fixed buccal appliances and removable aligners over one year of active orthodontic therapy. Angle Orthod 2013;83(1):146-51.

[20] Liu H, Sun J, Dong Y, et al. Periodontal health and relative quantity of subgingival porphyromonas gingivalis during orthodontic treatment. Angle Orthod 2011;81(4):609-15.

[21] Naranjo AA, Triviño ML, Jaramillo A, et al. Changes in the subgingival microbiota and periodontal parameters before and 3 months after bracket placement. Am J Orthod Dentofacial Orthop 2006;130(3):275.e17-22.

[22] Sharma K, Mangat S, Kichorchandra MS, et al. Correlation of orthodontic treatment by fixed or myofunctional appliances and periodontitis: a retrospective study. J Contemp Dent Pract 2017;18(4):322-5.

[23] Ireland AJ, Soro V, Sprague SV, et al. The effects of different orthodontic appliances upon microbial communities. Orthod Craniofac Res 2014;17(2):115-23.

[24] Kim SH, Choi DS, Jang I, et al. Microbiologic changes in subgingival plaque before and during the early period of orthodontic treatment. Angle Orthod 2011;82(2):254-60.

[25] Papageorgiou SN, Xavier GM, Cobourne MT, et al. Effect of orthodontic treatment on the subgingival microbiota: a systematic review and meta-analysis. Orthod Craniofac Res 2018;21(4):175-85.

[26] Pongnaratorn P, Kuacharan P, Kotsuno V, et al. In vitro antimicrobial activity of antidesma bunius extracts on oral pathogenic bacteria. Thai J Pharm Sci 2017;41(4):144-9.

[27] Veloso DJ, Abrão F, Martins CHG, et al. Potential antibacterial and anti-halitosis activity of medicinal plants against oral bacteria. Arch Oral Biol 2020;110:104585.
[28] Shah SS, Nambiar S, Kamath D, et al. Comparative evaluation of plaque inhibitory and antimicrobial efficacy of probiotic and chlorhexidine oral rinses in orthodontic patients: a randomized clinical trial. Inter J Dentistry 2019;2019:1-6.

[29] Dehghani M, Abtahi M, Hasanzadeh N, et al. Effect of propolis mouthwash on plaque and gingival indices over fixed orthodontic patients. J Clin Exp Dent 2019;11(3):e244-9.

[30] O'Reilly MM, Featherstone JD. Demineralization and remineralization around orthodontic appliances: an in vivo study. Am J Orthod Dentofacial Orthop 1987;92(1):33-40.

[31] Perrini F, Lombardo L, Arreghini A, et al. Caries prevention during orthodontic treatment: in-vivo assessment of high-fluoride varnish to prevent white spot lesions. Am J Orthod Dentofacial Orthop 2016;149(2):238-43.

[32] Singh S, Singh SP, Goyal A, et al. Effects of various remineralizing agents on the outcome of post-orthodontic white spot lesions (WSLs): a clinical trial. Prog orthod 2016;17(1):25.

[33] Yadav S, Sachdev V, Malik M, et al. Effect of three different compositions of topical fluoride varnishes with and without prior oral prophylaxis on Streptococcus mutans count in biofilm samples of children aged 2-8 years: a randomized controlled trial. J Indian Soc Pedod Prev Dent 2019;37(3):286-91.

[34] Maxfield BJ, Hamdan AM, Tüfekçi E, et al. Development of white spot lesions during orthodontic treatment: perceptions of patients, parents, orthodontists and general dentists. Am J Orthod Dentofacial Orthop 2012;141(3):337-44. 